\title{
The impact of modified exercise and relaxation therapy on chronic lower back pain in office workers: a randomized clinical trial
} \author{
Lee Ingle ${ }^{5}$, Brandon S. Shaw ${ }^{6}$, Ina Shaw ${ }^{6}$ \\ ${ }^{1}$ Sports Medicine Research Center, Neuroscience Institute, Tehran University of Medical Sciences, Tehran, Iran \\ 2Department of Anesthesiology, AJA University of Medical Sciences, Tehran, Iran \\ ${ }^{3}$ Department of Orthotics and Prosthetics, Iran University of Medical Sciences, Tehran, Iran \\ ${ }^{4}$ Department of Physiotherapy, School of Rehabilitation, Tehran University of Medical Sciences, Tehran, Iran \\ ${ }^{5}$ Department of Sport, Health \& Exercise Science, University of Hull, Kingston-upon-Hull, UK \\ ${ }^{6}$ Department of Human Movement Science, University of Zululand, KwaDlangezwa, South Africa
}

Ardalan Shariat ${ }^{1, *}$, Reza Alizadeh² ${ }^{2}$ Vahideh Moradi ${ }^{3}$, Elahe Afsharnia', Azadeh Hakakzadeh' ${ }^{1}$, Noureddin Nakhsotin Ansari ${ }^{1 / 4}$,

This study aimed to evaluate the effectiveness of a modified package of exercise therapy combined with relaxation on pain intensity, range of motion (ROM), anxiety, and quality of life ( $\mathrm{O}$ oL) in office workers with chronic lower back pain. In this clinical trial, 72 office workers aged 20 to 50 years with chronic low back pain were randomized to one of four groups including; group 1, exercise therapy; group 2, psychotherapy (relaxation therapy); group 3, modified protocol (exercise therapy followed by relaxation therapy); group 4, control group (no intervention). Participants exercised 3 times weekly for 6 weeks for $40-45$ min of exercise/relaxation. Pain intensity, ROM, anxiety, and QoL were evaluated at baseline, 6 weeks, and 12 weeks after the end of the intervention. Results showed significant decreases in pain intensity and anxiety in the three experimental groups compared to the control group. The three in- tervention groups indicated a significant decrease in pain intensity and anxiety after 6 and 12 weeks. ROM significantly improved in the exercise therapy and the modified protocol over time (after 6 and 12 weeks). A significant difference in $\mathrm{OoL}$ was found between the groups, with the greatest improvement found in the psychotherapy and modified protocol groups. This therapeutic package (including exercise movements and psychological interventions) was found to have a superior effect on pain intensity, ROM, anxiety, and QoL after 6 and 12 weeks compared to other interventions (only exercise and psychotherapy).

Keywords: Exercise therapy, Psychological, Low back pain, Office workers

\section{INTRODUCTION}

Lower back pain (LBP) is a common condition which impacts 85\%-90\% of people in both developed and developing countries (Asadi et al., 2016; Mehrdad et al., 2016; Mohseni-Bandpei et al., 2017). Most people have experienced LBP at least once in their lives, especially the nonspecified type defined as any low back pain in the lumbar area, which is not related to a severe pathology and is caused by unknown reasons (Maher et al., 2017; Synnott et al., 2015). If individuals do not take their LBP seriously, the pain may continue, intensify, and may ultimately lead to disability (O'Sullivan et al., 2019). The high prevalence of lumbar-related injuries can have a high impact at the individual level but also a significant impact on healthcare services locally, regionally and nationally (Natour et al., 2015; Richmond et al., 2015).

Due to prolonged sitting and inactivity in office workers, LBP is a common cause of disability (Matsudaira et al., 2012). The economic burden of LBP is exponential and driven by the loss of productivity and early retirement (Shariat et al., 2017). Therefore, understanding and identifying the factors that increase the risk of
${ }^{*}$ Corresponding author: Ardalan Shariat (D https://orcid.org/0000-0003-4750-5797 Sports Medicine Research Center, Neuroscience Institute, Tehran University of Medical Sciences, Tehran 11489, Iran

E-mail: ardalansh2002@gmail.com

Received: August 20, 2019 / Accepted: September 18, 2019
This is an Open Access article distributed under the terms of the Creative Commons Attribution Non-Commercial License (http://creativecommons.org/licenses/by-nc/4.0/) which permits unrestricted non-commercial use, distribution, and reproduction in any medium, provided the original work is properly cited. 
LBP are necessary, while developing effective rehabilitation strategies for work-related LBP (Steffens et al., 2016). Research has shown that LBP is a multifactorial disorder with several physical and psychological risk factors. Physical factors include stature, body mass, age and body mass index. Psychological factors include mental fatigue, anxiety and depression (Stubbs et al., 2016; Yang et al., 2016). In this regard, workplace stress can cause the lower back muscles to spasm, and increase the incidence of LBP. This is exacerbated by prolonged periods of sitting and general sedentariness in office workers (Hurwitz et al., 2005; Stubbs et al., 2016; Synnott et al., 2016). Hence, both the physical and psychological wellbeing of an individual with LBP needs to be addressed in the management of LBP. Recently, new treatment methods based on physical exercise have been proposed, but these methods generally focus on the full length of the spine (Miyamoto et al., 2019; Shariat et al., 2018). Often the impact of psychological wellbeing is not included in interventions. Therefore, the aim of the current study is to modify a new therapeutic exercise protocol presented by a recent research in 2017 (Shariat et al., 2018) with the purpose of representing an intervention that addresses both physical and psychological wellbeing.

\section{MATERIALS AND METHODS}

The present study was a single-blind randomized control trial with parallel groups. In this study, office workers with chronic LBP were selected. Following ethical approval (IRCT20171111 037388N2, Tehran University of Medical Sciences), participants provided full written consent prior to participation. The inclusion criteria for enrollment was class 11, I American Society of Anaesthesiologists physical status classification, aged 20-50 years, chronic LBP for more than 3 months with at least two symptoms of chronic LBP including pain reported when lifting heavy loads or feeling pain during changing postural position, absence of pain in lower limb during physical testing, absence of disc tearing, presence of low intensity pain signals between vertebral area, and absence of joint burst following magnetic resonance imagining (MRI). The participants were excluded if they used corticosteroids or had symptoms (dizziness or unconscious feeling, paralysis) prior to the start of the study. Each participant was evaluated by a qualified physician and underwent an MRI scan to determine the extent of damage in the lower back region.

Based on the randomized block design, participants were randomly assigned into one of four parallel study arms; group 1, exercise therapy; group 2, psychotherapy (relaxation therapy); group
3 , modified protocol (exercise therapy followed by relaxation therapy); group 4, control group (no intervention). Physical exercises and relaxation techniques were shown to clinical staff via an instructional video. Participants were required to complete three nonconsecutive sessions per week for 6 weeks, for $40-45$ minutes for each training session.

The physical exercise component included 13 simple exercise movements that had to be performed 3 times a day for 10 repetitions. The exercises were derived from Williams and McKenzie and followed the recommendations of the American College of Sports Medicine. This protocol was previously introduced by Shariat et al. (2017) as an effective protocol for LBP. The relaxation component was based on the stress management model, and followed guidance provided by Conrad et al. (2007). To perform this test, participants were instructed to relax for 8 min while they sat on a comfortable chair in a large room with appropriate light and temperature without any noise. Participants were then asked to breathe at their usual depth and rate and then for 4 min they were required to inhale for a count of 10 and to exhale for a count of 10 while thinking of the word "relax." During the test their eyes were open and they were asked to breathe through their nose.

The modified protocol required the participants to perform the relaxation training for 20-25 minutes. Each session was led by a sports psychologist. This was followed by the physical exercise component (Conrad et al., 2007).

\section{Outcome measurements}

The outcome measurements included the evaluation of quality of life (QoL) using the QoL questionnaire (Burckhardt and Anderson, 2003), anxiety and depression using the hospital anxiety and depression (HADS) questionnaire (Montazeri et al., 2003), the severity of pain based on the Functional Rating Index test (Ceran and Ozcan, 2006) and the hips' range of motion (ROM) using the Thomas test before the treatment, at 6 weeks after the end of the intervention, and 12 weeks following the intervention. Cronbach alpha of the Iranian HADS questionnaire was obtained between 0.78 and 0.86 for anxiety and depression, respectively.

\section{Sample size}

The sample size was calculated separately for each dependent variable, and the highest number was considered as the sample size for our study. The sample size for each group was 28 participants with an additional $20 \%$ added due to likely attrition over the 6-week intervention. As such, 34 participants were required for each group. 


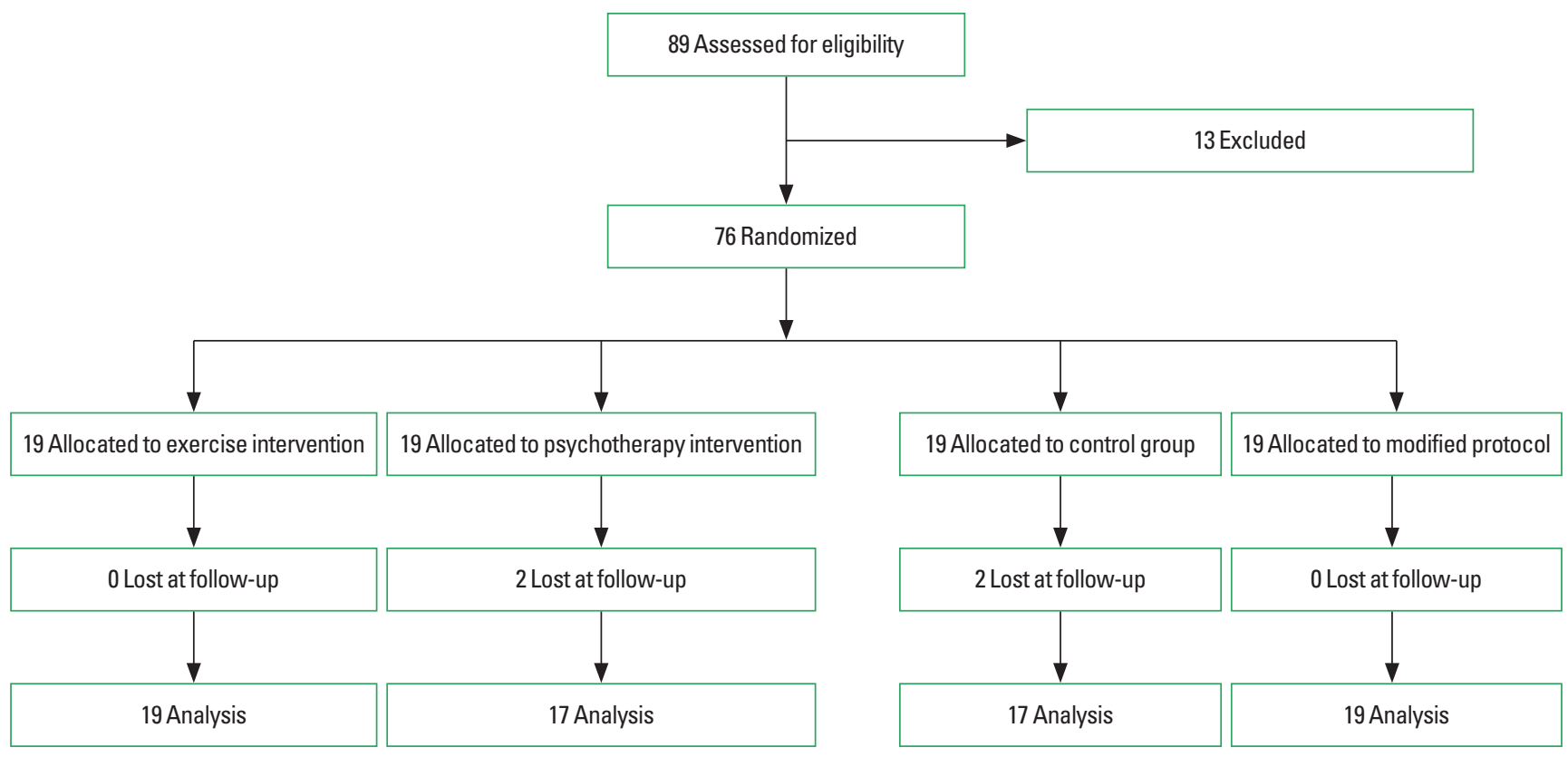

Fig. 1. Sampling framework of the study.

\section{Data analysis}

To evaluate the normality of the data, a Kolmogorove-Smirnove test was performed. The distribution of all of the variables was normal. After evaluating the normality of data; ranges, means, and standard deviations were reported and baseline characteristics between groups were assessed by the independent $t$-test. For each variable the main and interactive effects of group and time were determined using a mixed model of analysis of variance. The data analysis was carried out using IBM SPSS Statistics ver. 22.0 (IBM Co., Armonk, NY, USA).

\section{RESULTS}

Of the 89 participants who volunteered for the study, 76 were eligible to participate. Of the initial 76 participants, 72 (95\%) completed the study. Four participants did not complete the study due to falling ill (Fig. 1).

No significant differences were found at baseline between the four groups. Data revealed a significant improvement in the ROM of the exercise therapy and modified protocol groups when compared to the control and the psychotherapy groups. The results showed that there was a significant group-by-time interaction effect and a main effect for time $(P<0.001)$. The Bonferroni test revealed a significant improvement in the ROM of the modified protocol after 6 and 12 weeks, but no significant effect between 6 and 12 weeks (Table 1).
Table 1. Study arm data for range of motion (ROM), pain, quality of life, and anxiety at each time point $(n=72)$

\begin{tabular}{lcccc} 
Variable & $\begin{array}{c}\text { Exercise } \\
\text { therapy }(n=19)\end{array}$ & $\begin{array}{c}\text { Psychotherapy } \\
(n=17)\end{array}$ & $\begin{array}{c}\text { Modified } \\
\text { protocol }(n=19)\end{array}$ & $\begin{array}{c}\text { Control } \\
(n=17)\end{array}$ \\
\hline ROM $\left(^{\circ}\right)$ & & & & \\
Baseline & $70 \pm 10$ & $68 \pm 9$ & $71 \pm 10$ & $72 \pm 7$ \\
6 Weeks & $73 \pm 9$ & $71 \pm 12$ & $74 \pm 11$ & $73 \pm 8$ \\
12 Weeks & $76 \pm 11$ & $73 \pm 10$ & $79 \pm 10$ & $73 \pm 7$ \\
P-value & 0.040 & 0.050 & 0.010 & 0.120 \\
Pain & & & & \\
Baseline & $13 \pm 2$ & $12 \pm 2$ & $14 \pm 1$ & $12 \pm 2$ \\
6 Weeks & $4 \pm 1$ & $5 \pm 1$ & $4 \pm 1$ & $11 \pm 2$ \\
12 Weeks & $3 \pm 2$ & $3 \pm 1$ & $2 \pm 1$ & $11 \pm 1$ \\
P-value & 0.020 & 0.030 & 0.020 & 0.130 \\
Quality of life & & & & \\
Baseline & $42 \pm 6$ & $46 \pm 4$ & $44 \pm 5$ & $19 \pm 7$ \\
6 Weeks & $64 \pm 4$ & $62 \pm 7$ & $64 \pm 6$ & $17 \pm 3$ \\
12 Weeks & $68 \pm 5$ & $66 \pm 8$ & $66 \pm 4$ & $18 \pm 4$ \\
P-value & 0.060 & 0.040 & 0.010 & 0.170 \\
Anxiety & & & & \\
Baseline & $14 \pm 2$ & $15 \pm 2$ & $14 \pm 3$ & $13 \pm 2$ \\
6 Weeks & $12 \pm 3$ & $11 \pm 2$ & $10 \pm 4$ & $11 \pm 2$ \\
12 Weeks & $8 \pm 1$ & $7 \pm 2$ & $6 \pm 3$ & $12 \pm 2$ \\
P-value & 0.010 & 0.040 & 0.010 & 0.170 \\
\hline
\end{tabular}

Values are presented as mean \pm standard deviation unless otherwise indicated.

The pain intensity in all intervention groups significantly decreased. The time effect was also significant in the all intervention groups. Post hoc analysis revealed that pain reduced after 6 and 12 
weeks $(P<0.001)$. However, no significant difference was found between 6 and 12 weeks (Table 1).

The QoL significantly increased after the psychotherapy and modified protocol groups compared with the exercise therapy and control groups. Group-by-time interaction effect and a main effect for time revealed an improvement in QoL after 6 and 12 weeks in the psychotherapy and modified protocol groups $(P<0.001)$. There was no significant difference between 6 and 12 weeks (Table 1).

Anxiety and depression decreased significantly in all three intervention groups compared to the control group. Also, there was a main effect of time on anxiety and depression. Post hoc analysis did not show any significant difference in anxiety and depression between weeks 6 and 12, but anxiety did significantly decrease after 6 and 12 weeks compared to baseline in all the intervention groups $(P<0.001)$ (Table 1$)$.

\section{DISCUSSION}

This study aimed to determine the effectiveness of a modified protocol of exercise therapy combined with psychotherapy on office workers suffering from LBP. Exercise therapy by itself and concurrent with relaxation is currently used in the treatment of patients with CLBP. It is considered as an effective therapy for increasing ROM. Improvement in ROM is attained due to increased blood flow to muscles and reduced stiffed joints (Gordon and Bloxham, 2016). The results indicated that the modified protocol and exercise therapy increase ROM. The effect of exercise on flexibility and ROM is well known since exercise results in the mechanical stretching of the tendons, intramuscular connective tissue and other passive skeletal structures (Kubo et al., 2001; Morse et al., 2008). This increase in ROM as a result of exercise is essential to improve or eliminate impairments in back flexibility that can result in alteration of the relaxation response of the paraspinal musculature associated with full spinal flexibility and shortening of muscles and connective tissues of the spinal region (Rainville et al., 2004). In addition, it was found that the effectiveness of these therapies lasts for 3 months after the therapy sessions have ended. The results of this study are in line with those of Gordon and Bloxham (2016), Freimann et al. (2015), and Kim et al. (2015) which similarly showed that multidimensional interventions are effective in the treatment of LBP. As such, interventions which focus on both the physical and mental components of pain can have positive effects, but previous published studies are limited in this field (Hoffman et al., 2007; Niknejad et al., 2018; Weisenberg, 1987).
The results showed that exercise therapy, psychotherapy, and the combination of both treatments significantly decreased the pain intensity in the three experimental groups compared to the control group. This indicates the effect of these interventions on the pain intensity of the office workers. In the study of Hayden et al. (2005) progressive resistance exercises, which strengthen the abdominal and spinal muscles are effective in reducing the pain in individuals with LBP. Also, this finding is consistent with that of Good who reported that psychotherapy (relaxation techniques) is effective in the treatment of employees with LBP (Good, 1995). Further, a study by Okhovatian et al. (2003) showed that exercise therapy using similar exercises to those prescribed in the present study revealed significant reductions in the pain intensity associated with LBP. While all intervention groups observed a reduction in pain, it is important to note that they would have done so via divergent analgesic pathways, while the combined treatment group may have benefited from both exercise and psychotherapy. Exercise may reduce pain via an enhanced blood flow, which is thought to help in dissipating algogenic chemicals, by facilitating muscle relaxation and activation of supraspinal pathways or by reducing peripheral edema by virtue of its effect on the cardiovascular system and by centrally dampening sympathetic nervous activity (Hall et al., 2008). It is important to note that psychotherapy does not directly address the physiological component of pain in LBP, but rather results in the reframing of maladaptive thoughts and coping strategies, which may, reduce the pain experience associated with LBP (Gatchel and Rollings, 2008).

The present study demonstrated an improvement in the QoL in the psychotherapy and modified protocol groups. The results of this study are in line with those of Tavafian who concluded that an educational program promoted the QoL of women suffering from a LBP (Tavafian et al., 2007). Although this study is different from the current study in terms of intervention type and research population, the results indicate that the QoL of individuals suffering chronic LBP can be affected by their mental issues (Horng et al., 2005). In the present study, it appears that psychotherapy, either alone or combination, improves QoL. Psychotherapy may have improved QoL due to its ability to expand the patients' repertoire of effective coping skills and by fostering realistic hope and optimism, while enhancing wellbeing (Courneya et al., 2003). While psychotherapy is the "gold standard" to improve QoL, it was disappointing that our exercise intervention did not produce improvements in QoL, since psychotherapeutic interventions are less likely to address the physical and functional problems encountered by patients with LBP, such as reduced cardio- 
pulmonary function and loss of muscle strength (Courneya et al., 2003), hence the need for a combined intervention.

The results of the present study showed a significant improvement in anxiety and depression in all the intervention groups. In line with the present study, Diepenmaat et al. (2006) showed that musculoskeletal pain is associated with depression and stress. Either psychotherapy or exercise alone was sufficient to improve anxiety and depression. The finding in this study and in previous studies that exercise is equally powerful to psychotherapy in improving anxiety and depression is essential in that many individuals avoid psychotherapy because of perceived stigma (Mead et al., 2009). While there is evidence that psychotherapy and exercise are effective in treating anxiety and depression alone, the mechanisms by which this occurs are unclear (i.e., treating the primary symptoms of depressive disorders or whether the effect was achieved through other mechanisms such as simple recovery from/ improvement in LBP) any future understanding of the mechanisms by which psychotherapy and exercise improve anxiety and depression may help to determine their potential overlapping or complementary nature (Byrne and Byrne, 1993).

A modified protocol utilizing a combination of exercise therapy and psychotherapy can improve pain, anxiety, ROM and $\mathrm{QoL}$ in office workers with LBP. In fact, this study confirmed that a multidisciplinary intervention is needed in the treatment of LBP in office workers.

\section{CONFLICT OF INTEREST}

No potential conflict of interest relevant to this article was reported.

\section{ACKNOWLEDGMENTS}

This study was supported and approved by Tehran University of Medical Sciences (IR.TUMS.VCR.REC.1396.4380).

\section{REFERENCES}

Asadi P, Monsef KV, Zia ZM, Zohrevandi B. The prevalence of low back pain among nurses working in Poursina hospital in Rasht, Iran. J Emer Prac Traum 2016;2:11-15.

Burckhardt CS, Anderson KL. The Quality of Life Scale (QOLS): reliability, validity, and utilization. Health Qual Life Outcomes 2003;1:60.

Byrne A, Byrne DG. The effect of exercise on depression, anxiety and other mood states: a review. J Psychosom Res 1993;37:565-574.
Ceran F, Ozcan A. The relationship of the Functional Rating Index with disability, pain, and quality of life in patients with low back pain. Med Sci Monit 2006;12:CR435-439.

Conrad A, Müller A, Doberenz S, Kim S, Meuret AE, Wollburg E, Roth WT. Psychophysiological effects of breathing instructions for stress management. Appl Psychophysiol Biofeedback 2007;32:89-98.

Courneya KS, Friedenreich CM, Sela RA, Quinney HA, Rhodes RE, Handman $\mathrm{M}$. The group psychotherapy and home-based physical exercise (group-hope) trial in cancer survivors: physical fitness and quality of life outcomes. Psychooncology 2003;12:357-374.

Diepenmaat AC, van der Wal MF, de Vet HC, Hirasing RA. Neck/shoulder, low back, and arm pain in relation to computer use, physical activity, stress, and depression among Dutch adolescents. Pediatrics 2006;117:412-416.

Freimann T, Merisalu E, Pääsuke M. Effects of a home-exercise therapy programme on cervical and lumbar range of motion among nurses with neck and lower back pain: a quasi-experimental study. BMC Sports Sci Med Rehabil 2015;7:31.

Gatchel RJ, Rollings KH. Evidence-informed management of chronic low back pain with cognitive behavioral therapy. Spine J 2008;8:40-44.

Good M. A comparison of the effects of jaw relaxation and music on postoperative pain. Nurs Res 1995;44:52-57.

Gordon R, Bloxham S. A systematic review of the effects of exercise and physical activity on non-specific chronic low back pain. Healthcare (Basel) 2016;4(2):E22.

Hall J, Swinkels A, Briddon J, McCabe CS. Does aquatic exercise relieve pain in adults with neurologic or musculoskeletal disease? A systematic review and meta-analysis of randomized controlled trials. Arch Phys Med Rehabil 2008;89:873-883.

Hayden JA, van Tulder MW, Tomlinson G. Systematic review: strategies for using exercise therapy to improve outcomes in chronic low back pain. Ann Intern Med 2005;142:776-785.

Hoffman BM, Papas RK, Chatkoff DK, Kerns RD. Meta-analysis of psychological interventions for chronic low back pain. Health Psychol 2007;26:1-9.

Horng YS, Hwang YH, Wu HC, Liang HW, Mhe YJ, Twu FC, Wang JD. Predicting health-related quality of life in patients with low back pain. Spine (Phila Pa 1976) 2005;30:551-555.

Hurwitz EL, Morgenstern H, Chiao C. Effects of recreational physical activity and back exercises on low back pain and psychological distress: findings from the UCLA Low Back Pain Study. Am J Public Health 2005;95:1817-1824.

Kim TH, Kim EH, Cho HY. The effects of the CORE programme on pain at rest, movement-induced and secondary pain, active range of motion, and proprioception in female office workers with chronic low 
back pain: a randomized controlled trial. Clin Rehabil 2015;29:653-662.

Kubo K, Kanehisa H, Kawakami Y, Fukunaga T. Influence of static stretching on viscoelastic properties of human tendon structures in vivo. J Appl Physiol (1985) 2001;91:277-282.

Maher C, Underwood M, Buchbinder R. Non-specific low back pain. Lancet 2017;389:736-747.

Matsudaira K, Konishi H, Miyoshi K, Isomura T, Takeshita K, Hara N, Yamada K, Machida H. Potential risk factors for new onset of back pain disability in Japanese workers: findings from the Japan epidemiological research of occupation-related back pain study. Spine (Phila Pa 1976) 2012;37:1324-1333.

Mead GE, Morley W, Campbell P, Greig CA, McMurdo M, Lawlor DA. Exercise for depression. Cochrane Database Syst Rev 2009;(3):CD004366.

Mehrdad R, Shams-Hosseini NS, Aghdaei S, Yousefian M. Prevalence of low back pain in health care workers and comparison with other occupational categories in iran: a systematic review. Iran J Med Sci 2016;41:467-478.

Miyamoto GC, Lin CC, Cabral CMN, van Dongen JM, van Tulder MW. Cost-effectiveness of exercise therapy in the treatment of non-specific neck pain and low back pain: a systematic review with meta-analysis. Br J Sports Med 2019;53:172-181.

Mohseni-Bandpei MA, Rahmani N, Halimi F, Farooq MN. The prevalence of low back pain in Iranian dentists: an epidemiological study. Pak J Med Sci 2017;33:280-284.

Montazeri A, Vahdaninia M, Ebrahimi M, Jarvandi S. The Hospital Anxiety and Depression Scale (HADS): translation and validation study of the Iranian version. Health Qual Life Outcomes 2003;1:14.

Morse CI, Degens H, Seynnes OR, Maganaris CN, Jones DA. The acute effect of stretching on the passive stiffness of the human gastrocnemius muscle tendon unit. J Physiol 2008;586:97-106.

Natour J, Cazotti Lde A, Ribeiro LH, Baptista AS, Jones A. Pilates improves pain, function and quality of life in patients with chronic low back pain: a randomized controlled trial. Clin Rehabil 2015;29:59-68.

Niknejad B, Bolier R, Henderson CR Jr, Delgado D, Kozlov E, Löckenhoff CE, Reid MC. Association between psychological interventions and chronic pain outcomes in older adults: a systematic review and meta-analysis. JAMA Intern Med 2018;178:830-839.

Okhovatian F, Kahrizi S, Samadi-Pour A. Comparison between three common remedial exercises in pain severity of patients with mechanical CLBP. J Rehab 2003;4:7-15.

O'Sullivan K, O'Keeffe M, Forster BB, Qamar SR, van der Westhuizen A, O'Sullivan PB. Managing low back pain in active adolescents. J Ma- nipulative Physiol Ther 2019;26:1-8.

Rainville J, Hartigan C, Martinez E, Limke J, Jouve C, Finno M. Exercise as a treatment for chronic low back pain. Spine J 2004;4:106-115.

Richmond H, Hall AM, Copsey B, Hansen Z, Williamson E, HoxeyThomas N, Cooper Z, Lamb SE. The effectiveness of cognitive behavioural treatment for non-specific low back pain: a systematic review and meta-analysis. PLoS One 2015;10:e0134192.

Shariat A, Cleland JA, Danaee M, Kargarfard M, Sangelaji B, Tamrin SBM. Effects of stretching exercise training and ergonomic modifications on musculoskeletal discomforts of office workers: a randomized controlled trial. Braz J Phys Ther 2018;22:144-153.

Shariat A, Lam ET, Kargarfard M, Tamrin SB, Danaee M. The application of a feasible exercise training program in the office setting. Work 2017; 56:421-428.

Steffens D, Maher CG, Pereira LS, Stevens ML, Oliveira VC, Chapple M, Teixeira-Salmela LF, Hancock MJ. Prevention of low back pain: a systematic review and meta-analysis. JAMA Intern Med 2016;176:199-208.

Stubbs B, Koyanagi A, Thompson T, Veronese N, Carvalho AF, Solomi M, Mugisha J, Schofield P, Cosco T, Wilson N, Vancampfort D. The epidemiology of back pain and its relationship with depression, psychosis, anxiety, sleep disturbances, and stress sensitivity: data from 43 low- and middle-income countries. Gen Hosp Psychiatry 2016;43: 63-70.

Synnott A, O'Keeffe M, Bunzli S, Dankaerts W, O'Sullivan P, O'Sullivan K. Physiotherapists may stigmatise or feel unprepared to treat people with low back pain and psychosocial factors that influence recovery: a systematic review. J Physiother 2015;61:68-76.

Synnott A, O'Keeffe M, Bunzli S, Dankaerts W, O'Sullivan P, Robinson K, O'Sullivan K. Physiotherapists report improved understanding of and attitude toward the cognitive, psychological and social dimensions of chronic low back pain after Cognitive Functional Therapy training: a qualitative study. J Physiother 2016;62:215-221.

Tavafian SS, Jamshidi A, Mohammad K, Montazeri A. Low back pain education and short term quality of life: a randomized trial. BMC Musculoskelet Disord 2007;8:21.

Weisenberg M. Psychological intervention for the control of pain. Behav Res Ther 1987;25:301-312.

Yang H, Haldeman S, Lu ML, Baker D. Low back pain prevalence and related workplace psychosocial risk factors: a study using data from the 2010 National Health Interview Survey. J Manipulative Physiol Ther 2016;39:459-472. 